\title{
Journal of

\section{Synthesis of mesoporous composite materials of nitrogen-doped carbon and silica using a reactive surfactant approach $\dagger$}

\author{
Jens Peter Paraknowitsch, ${ }^{* a}$ Yuanjian Zhang $^{b}$ and Arne Thomas ${ }^{a}$ \\ Received 15th April 2011, Accepted 2nd August 2011 \\ DOI: $10.1039 / c 1 j m 11633 a$
}

\begin{abstract}
Mesoporous composite materials of nitrogen-doped carbon and silica were synthesised in a one-stepprocess applying a soft templating procedure. The template used in the sol-gel synthesis of the silica is a cationic surfactant with distinct reactivity to form nitrogen-doped graphitic carbon upon heating. This reactivity is derived from the combination of the dicyanamide anion with a nitrogen-containing pyridinium cation, as it is known from ionic liquids used as nitrogen-doped carbon precursors. Thus applying this surfactant in a conventional sol-gel synthesis yields a silica gel doped with a precursor for $\mathrm{N}$-doped carbon. By subsequent annealing mesoporous composite materials of silica and nitrogendoped carbon are obtained.
\end{abstract}

\section{Introduction}

Nitrogen-doped carbon materials have raised major interest in the past few years of research due to their promising performance in various applications. Oxygen reduction reaction catalysis, ${ }^{1-5}$ anode materials in lithium ion batteries, ${ }^{6-9}$ electrodes in electrochemical double layer capacitors ${ }^{10-14}$ or sequestration of carbon dioxide $^{15-17}$ are some examples showing that research focusing on nitrogen-doped carbon materials exhibits a significant relevance.

Nitrogen doped carbons are most frequently synthesised via thermolytic treatment of different nitrogen-containing precursors or precursor mixtures, such as polymers, aminated sugars or heterocycles, ${ }^{18-21}$ or by post-treatment of carbonaceous substrates with a reactive nitrogen source, e.g. ammonia or urea. $^{22,23}$ Recently we have been able to present a novel type of synthesis route, using ionic liquids composed of nitrogen containing cations and cyano functionalised anions, dicyanamide (dca), as precursors in a facile, one-step synthesis. ${ }^{24,25}$ The resulting materials showed promising properties: nitrogen is structurally incorporated into the graphitic domains of the turbostratic material in the form of pyridinic or quaternary graphitic nitrogen. Thus the material exhibits good electric conductivity and significant stability against oxidation. Studies of other groups have been focusing on related concepts. ${ }^{26-29}$ To make the materials more interesting for potential applications there are two requirements to be fulfilled: nanoscaled

${ }^{a}$ Berlin Technical University, Institute of Chemistry-Functional Materials, Englische Str. 20, 10587 Berlin, Germany. E-mail: jens.p. paraknowitsch@tu-berlin.de; Fax: +49 (0)30 314 29271; Tel: +49 (0)30 31425241

${ }^{b}$ National Institute for Material Science, ICYS-MANA, 1-1 Namiki, Tsukuba, 305-0044, Japan

$\uparrow$ Electronic supplementary information (ESI) available. See DOI: $10.1039 / \mathrm{c} 1 \mathrm{jm} 11633 \mathrm{a}$ morphologies for high active surface areas should be introduced and the high costs of the materials have to be reduced. This can be realised by designing a composite material consisting of a common nanostructured support material functionalised with nitrogen-doped carbon. Therefore in this study we were focusing on the preparation of a mesoporous silica material coated with a layer of nitrogen-doped carbon.

Furthermore, ionic liquids are known to be highly suitable for the synthesis of different inorganic materials, as it has recently been reviewed by Dai et al. ${ }^{30}$ Among other examples, deep eutectic solvents, a supramolecular type of ionic liquids, have been applied successfully in the synthesis of carbon materials. ${ }^{31,32}$ The favourable interaction of the liquid salts with the polar inorganic structures gave rise to the synthesis of e.g. titania nanocrystals ${ }^{33}$ or hollow microspheres, ${ }^{34}$ mesoporous silica, ${ }^{35}$ photoluminescent $\mathrm{EuF}_{3}$ nanospheres ${ }^{36}$ and other functional nanostructures. ${ }^{37}$ Besides that, in our own studies the concept was proven in the synthesis of mesoporous nitrogen-doped carbon materials via different hard-templating approaches. ${ }^{24,25} \mathrm{It}$ is possible to further exploit this favourable interaction between polar inorganic materials and ionic liquids, using these properties for the direct formation of a nitrogen-doped carbon functionalised mesoporous silica material.

\section{Results and discussion}

In this work we combined the two concepts of soft templating ${ }^{38-40}$ and reactive templating. ${ }^{41-44}$ Therefore a novel type of surfactant was applied: $N$-hexadecyl-pyridinium-dicyanamide (NHP-dca) that could be synthesised easily by anion metathesis from the bromide derivative. ${ }^{45}$ This surfactant (shown in Fig. 1) combines the structure directing properties of a comparable cationic surfactant, such as cetyl-trimethyl-ammonium-bromide (CTAB), and the reactivity of the ionic liquids used for the synthesis of 


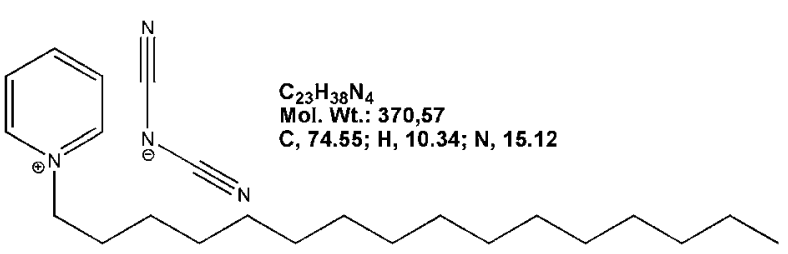

Fig. 1 Molecular formula of $N$-hexadecyl-pyridinium-dicyanamide (NHP-dca).

nitrogen-doped carbon. We are thus using a bifunctional surfactant that is not only capable of inducing mesostructures, but at the same time acts as a precursor for advanced chemical modifications of the targeted materials.

Mesoporous silica was synthesised by the controlled condensation of tetraethyl-orthosilicate (TEOS) in a basic aqueous solution of NHP-dca, varying the concentration of the surfactant and ammonia. The silica gels obtained by precipitation contain NHP-dca; subsequent thermal treatment of the gel in an inert gas atmosphere at $1000{ }^{\circ} \mathrm{C}$ yields the formation of nitrogen-doped carbon by thermolysis of the surfactant.

Experimental tests have shown that reaction mixtures composed of 1.2-1.6 mmol of NHP-dca, $15 \mathrm{~g}$ of deionised water, $1.20 \mathrm{~g}$ of a $25 \%$ aqueous ammonia solution and $6 \mathrm{mmol}$ of TEOS yield best results. Lower concentrations of NHP-dca did not yield sufficient formation of mesostructures, while higher concentrations did not yield porosity, probably due to blocking of the pores by an excess of carbonaceous material. Further, varying the $\mathrm{pH}$ changed the precipitation kinetics drastically; the amount of aqueous ammonia solution chosen that had been added to the system was found to be the most appropriate one for a controlled formation of the mesoporous silica gel.

The products obtained after annealing, synthesised with varying amounts of the surfactant $(1.2 \mathrm{mmol} / 1.4 \mathrm{mmol} /$ $1.6 \mathrm{mmol})$, are homogeneously black powders with increasing contents of $\mathrm{N}$-doped carbon (compare thermogravimetric data in Fig. 2).

The quantitative composition of the materials could be additionally analysed by X-ray photoelectron spectroscopy (XPS). The three elements carbon, nitrogen and silicon are detected as expected. Detailed scans of the energy regions according to $\mathrm{C} 1 \mathrm{~s}$,

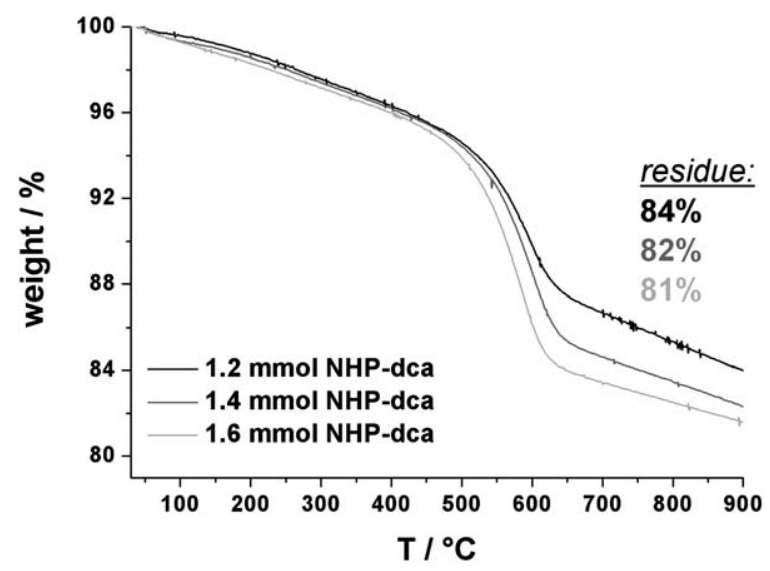

Fig. 2 TGA-curves; measurements accomplished under oxygen with a heating rate of $10 \mathrm{~K} \mathrm{~min}^{-1}$.

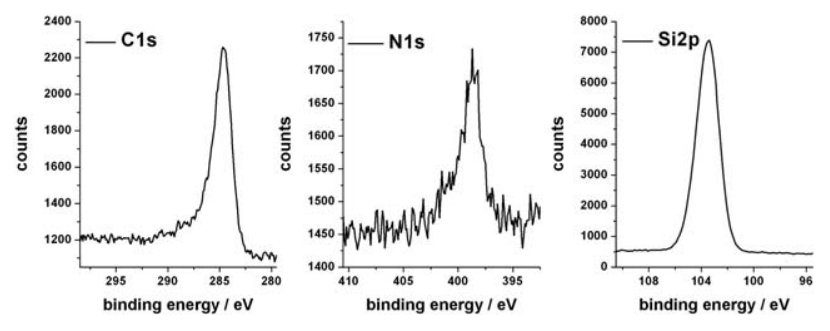

Fig. 3 Detailed XPS scans for C1s, N1s and Si2p orbitals. Results are shown for a sample obtained from highest concentration of the surfactant in the reaction mixture.

N1s and Si2p orbitals, respectively, are shown in Fig. 3 for the sample synthesised using $1.6 \mathrm{mmol}$ NHP-dca.

The quantitative evaluation of these data reveals an atomic N/C-ratio of $\sim 1 / 9$, which is in accordance with the nitrogen content known for the bulk nitrogen-doped carbon material. ${ }^{23,24}$ Identical results have been found for the samples derived from lower concentrations of NHP-dca, as it can be seen in Fig. S1 and $\mathrm{S} 2 \uparrow$. In this context it is interesting to note that the prolonged aliphatic chain does not significantly alter the elemental composition of the $\mathrm{N}$-doped carbon, as it is most likely to decompose during the carbonisation reaction. As XPS is a surface sensitive method, there is no evidence in deriving the entire elemental composition of the material from these data. Nevertheless it can be concluded that the nitrogen-doped carbon is successfully incorporated onto the silica surface. Furthermore it is possible to analyse the bonding environments of the different elements by deconvoluting the spectra. Thus silicon is-as expected - only found in one single valence state; the peak at $103.5 \mathrm{eV}$ (see Fig. $\mathrm{S} 3 \dagger$ ) refers to $\mathrm{SiO}_{2}$, which is supported by previous studies. ${ }^{46,47}$ The data for the $\mathrm{C} 1 \mathrm{~s}$ and the N1s orbitals reveal interesting details about the chemical composition of the $\mathrm{N}$-doped carbon in the mesoporous composite materials.

Fig. 4 shows the deconvoluted spectra of the $\mathrm{C} 1 \mathrm{~s}$ orbital. It shows a dominant contribution of graphitic carbon, with an intensity maximum at a binding energy of $284.6 \mathrm{eV} .^{47-49}$ There is further a minor contribution at $285.7 \mathrm{eV}$, which was reported to represent carbon bound to nitrogen atoms. ${ }^{50,51}$ Besides it cannot be entirely excluded contributions of contaminations by oxidised or other residual carbon species. ${ }^{47}$ Accordant evaluations of the scan for the N1s orbital (see Fig. 5) show that nitrogen is mainly found in a pyridinic bonding environment (398.7 eV), with

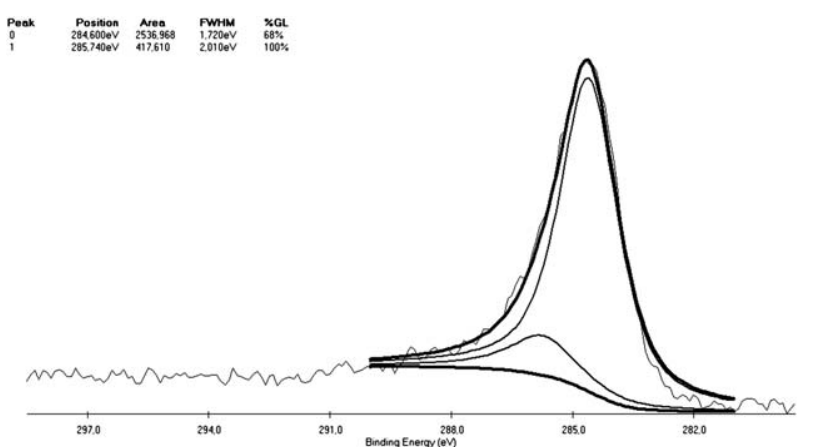

Fig. 4 Deconvoluted XPS-spectra of the C1s orbital, measured on a sample synthesised with $1.6 \mathrm{mmol}$ of NHP-dca. 


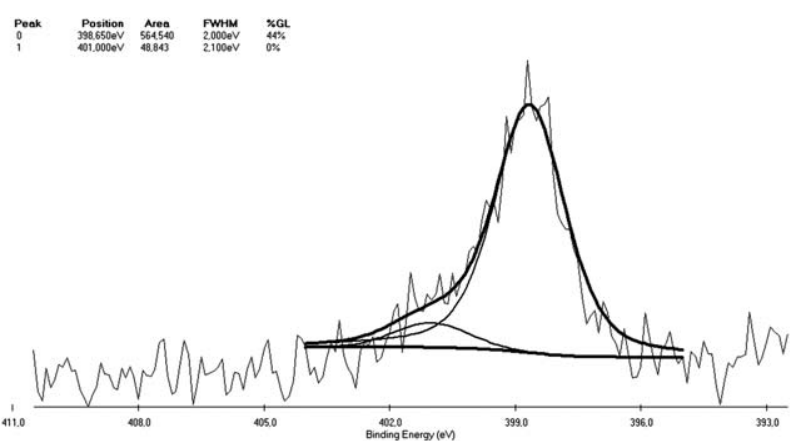

Fig. 5 Deconvoluted XPS-spectra of the N1s orbital, measured on a sample synthesised with $1.6 \mathrm{mmol}$ of NHP-dca.

minor contributions of quaternary graphitic nitrogen $(401.0 \mathrm{eV}) .^{23,47,52,53}$ This shows that the nitrogen is located dominantly at the edges of aromatic carbon layers, and that it is also directly incorporated into their structure. Contrarily the chemical environment of the nitrogen atoms in the bulk N-doped carbon materials derived from ionic liquid precursors is mainly quaternary graphitic nitrogen, with only minor contributions from pyridinic nitrogen..$^{24,25}$ This is due to the enhanced active surface area of the $\mathrm{N}$-doped carbon in the composite material (that will be discussed later throughout this manuscript), which increases the statistic probability of the placement of nitrogen atoms at the edges of graphitic layers.

Regarding the morphologies of the composite materials, high active surface areas of $994 \mathrm{~m}^{2} \mathrm{~g}^{-1}, 830 \mathrm{~m}^{2} \mathrm{~g}^{-1}$ and $717 \mathrm{~m}^{2} \mathrm{~g}^{-1}$, respectively, could be determined by nitrogen-sorption measurements (Fig. 6A). The surface area is slightly decreasing upon increasing the surfactant concentration. This is due to the higher amount of nitrogen-doped carbon in the composites. Furthermore, XRD patterns in the small angle range show broadened peaks indicating some degree of order of the porous structure (Fig. 6B).

A closer look at Fig. 6 reveals detailed information about the porous structure of the nitrogen-doped carbon coated silica. The diffraction lines in the XRD patterns are sharper for the materials synthesised at higher concentrations of the reactive surfactant. Thus it can be assumed that the higher concentrations yield more ordered structures. Further the hystereses of the nitrogen sorption isotherms are more pronounced for these materials, nevertheless they are still relatively broad and do not represent the significant region of the porous structure. This can be seen in the high slope in the lower relative pressure region between $p / p_{0}$ $=0$ and $p / p_{0}=0.3$ showing a strong contribution of pores in the smaller range of mesoporosity, while the slope is not steep enough for micropores. This is supported by a non-linear density functional theory (NLDFT) that has been applied for detailed pore size analysis. Thus pore size distributions indeed show defined pore diameters in the mesoporous region between $2 \mathrm{~nm}$ and $4 \mathrm{~nm}$, as it can be seen in Fig. 7 .

There is a tendency towards bimodal pore size distributions upon increasing the concentration of the surfactant. While samples synthesised with $1.2 \mathrm{mmol}$ NHP-dca only exhibit one maximum in their pore size distribution with a pore diameter of $2.5 \mathrm{~nm}$, increased surfactant concentration leads to a more pronounced contribution of pores with a diameter of $3.8 \mathrm{~nm}$.
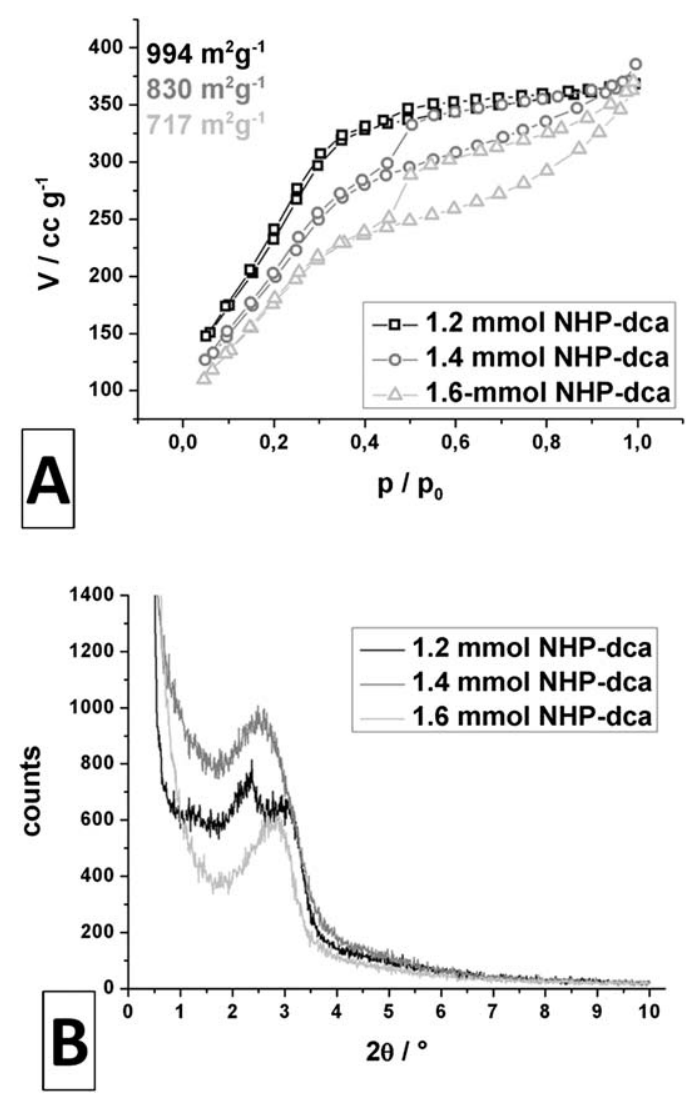

Fig. 6 (A) Nitrogen sorption isotherms of the N-doped carbon coated mesoporous silica. (B) Respective XRD patterns in the small angle range.

Nevertheless the pore size distributions remain narrow and are not broadened by this effect.

Furthermore, the morphology of the porosity of the different $\mathrm{N}$-doped carbon coated silica materials can be seen in the transition electron microscopy (TEM) micrographs represented in Fig. 8.

On the one hand it can be seen that the pores tend to be more ordered when applying higher concentrations of NHP-dca, while nevertheless it has to be stated that Fig. 8A cannot be perfectly compared to $\mathrm{B} / \mathrm{C}$ because there seem to be more layers of materials covering each other which slightly disproves the analysis of the porous structure. Still it is clearly pointed out the well defined order of the pores for higher concentrations of the surfactant. On the other hand the material is composed of submicron sized particles in which the small mesopores are located. Such or related particular morphologies of mesoporous silica have been frequently described when cationic surfactants are used in the synthetic procedure ${ }^{54-57}$ The spherical morphology can be identified easier by scanning electron microscopy (SEM), the accordant micrographs are represented in Fig. 9.

Nitrogen sorption isotherms, XRD, TEM and SEM enabled us to present an overview of the morphology of the materials under discussion: the pores are found in spherical particles, while pore diameters lie in the lower mesoporous region. Their order is increasing when using higher concentrations of NHP-dca upon synthesis. Further, a tendency towards bimodal pore size distributions is found for increasing concentrations of the surfactant. 

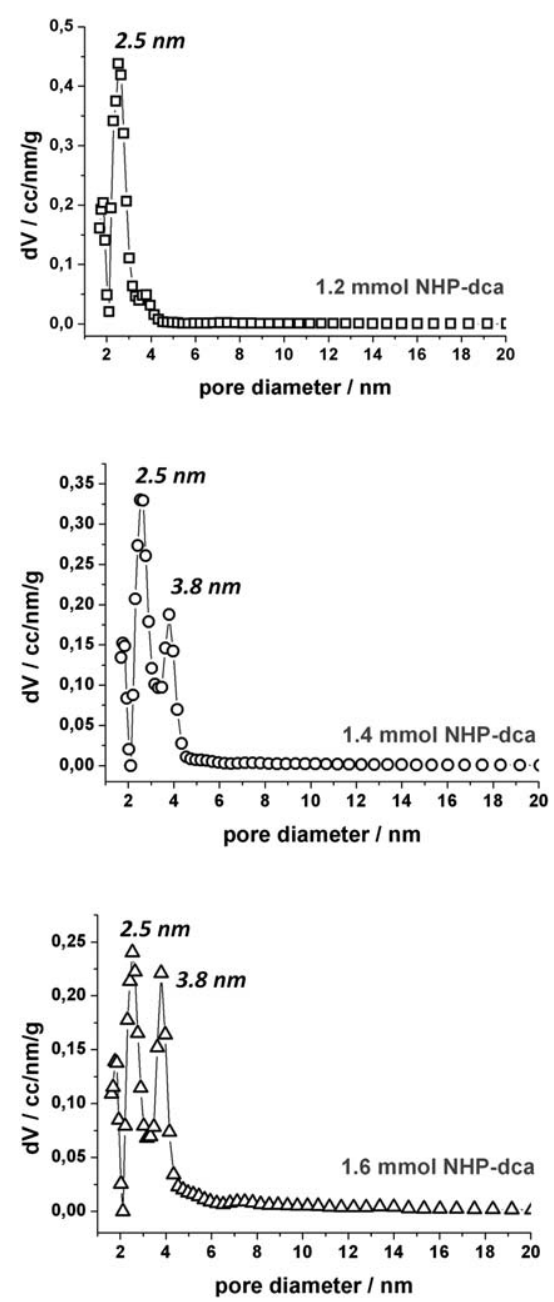

Fig. 7 NLDFT pore size distributions of the N-doped carbon coated mesoporous silica.

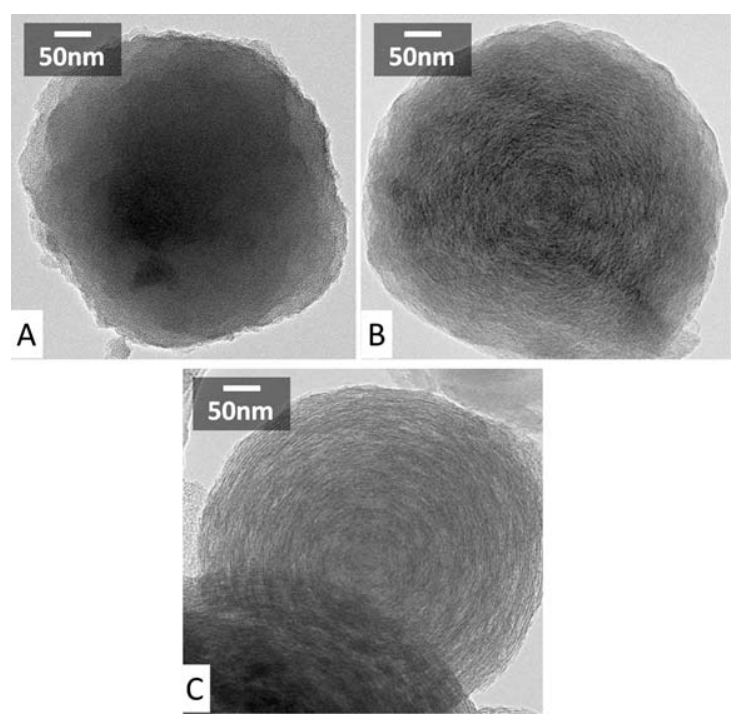

Fig. 8 TEM micrographs of the porous N-doped carbon coated silica, synthesised at different concentrations of NHP-dca: (A) $1.2 \mathrm{mmol}$, (B) $1.4 \mathrm{mmol}$, and (C) $1.6 \mathrm{mmol}$.

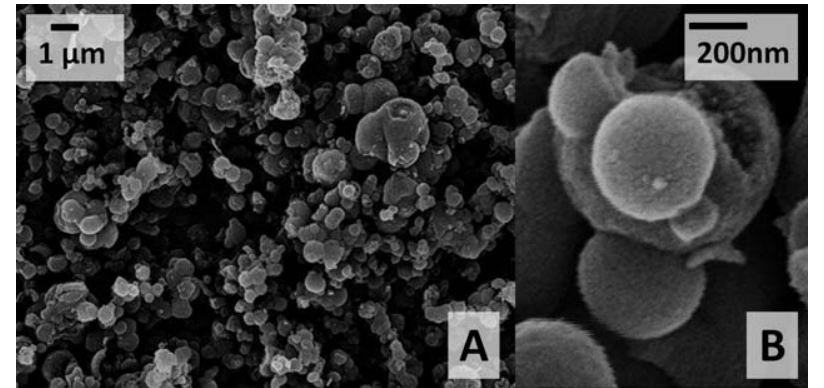

Fig. 9 Scanning electron micrographs (exemplarily for $1.6 \mathrm{mmol}$ NHPdca): (A) overview showing spherical particles and (B) close-up, porous structure of spheres is recognisable.

To understand this latter phenomenon it is important to distinguish between a possible influence of the composite formation and an influence of the surfactant itself on this kind of pore morphology. Therefore silica gels have been calcined under air directly after precipitation, so that pure mesoporous silica is obtained instead of a composite of $\mathrm{N}$-doped carbon and silica. The products have thus been characterised by nitrogen-sorption measurements. Fig. 10 is representing all the respective isotherms, including the active surface areas according to BET evaluations. The pore size distributions that were derived from these isotherms are shown in detail in the ESI, Fig. S4-S6†.

The surface area is higher than for the composite materials, while the shape of the isotherm is mainly the same. The isotherms are rather shifted to higher adsorption volumes. Furthermore the pore size distributions (Fig. S4-S6†) show a slight increase of the pore diameters, as the materials are pure mesoporous silicas, without the presence of nitrogen-doped carbon as a coating on the pore walls. These data further enable rough estimations of the thickness of the carbonaceous layer of $<1 \mathrm{~nm}$. As well for these materials a tendency towards bimodal pore size distributions is observed upon increasing the amount of surfactant used. This shows that the bimodal pore size distributions are induced directly by the structure directing properties of NHP-dca during the sol-gel synthesis of silica. The formation of the composite with the $\mathrm{N}$-doped carbon is thus not the driving force for the bimodality. It can be summed up that the $\mathrm{pH}$ value of the reaction medium and the concentration of the reactive surfactant are the crucial parameters for adjusting the properties of the obtained materials. While the $\mathrm{pH}$ value mainly defines the feasibility of the synthesis, the concentration of NHP-dca is directly influencing the porosity of the mesoporous composite materials.

Although - as discussed before-the carbonisation and composite formation are not found to be responsible for the formation of bimodal pore size distributions, the carbonisation of the reactive surfactant in the pores of the silica impacts the structure of the silica, as it can be derived from the isotherms measured on the pure silica materials that have been obtained by calcination of the samples after the formation of the composites. After removal of the carbon, the active surface areas of the materials are reduced, and the pore diameters are either not changed or slightly shifted towards smaller values (compare Fig. S7-10†), while the tendency to bimodal pore size distributions remains. It is most likely that the porous structure is at least 

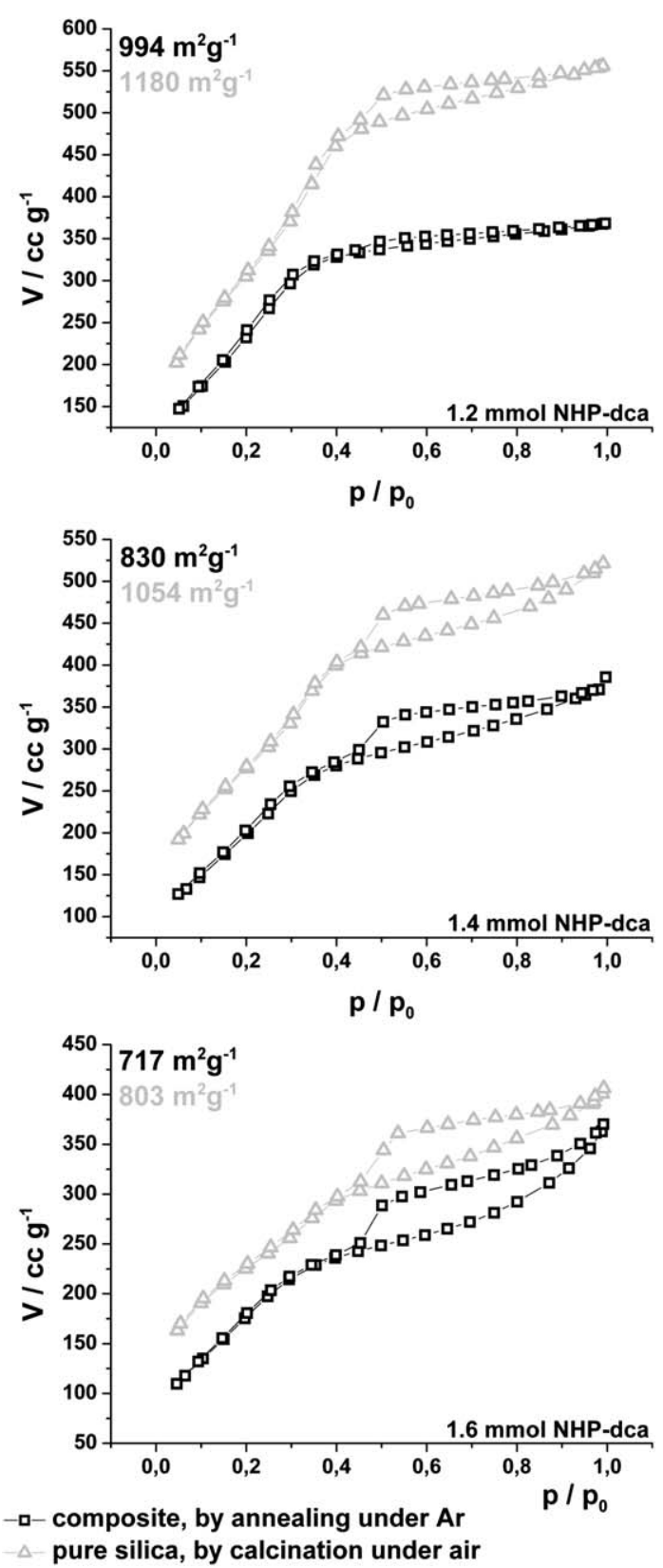

Fig. 10 Nitrogen-sorption isotherms of composite and pure silica materials, synthesised with varied amounts of NHP-dca surfactant.

partially collapsing due to the exhaustive thermal treatment. This can be additionally shown by measurements on the pure carbonaceous component after removal of the silica (exemplarily for the synthesis approach with $1.6 \mathrm{mmol}$ NHP-dca).

The respective nitrogen sorption isotherm (Fig. 11A) shows that the carbon does not exhibit any specific order. Just a relatively high active surface area of $467 \mathrm{~m}^{2} \mathrm{~g}^{-1}$ is maintained, caused by a templating effect of the silica support. The open shape of the isotherm indicates that the as obtained $\mathrm{N}$-doped carbon is a rather soft material, which also leads to a collapse of the structure. This can be seen in Fig. 11B: in the TEM micrograph the spherical morphology of the material is still observed, but the collapsed character of the porosity is clearly visible. It is thus

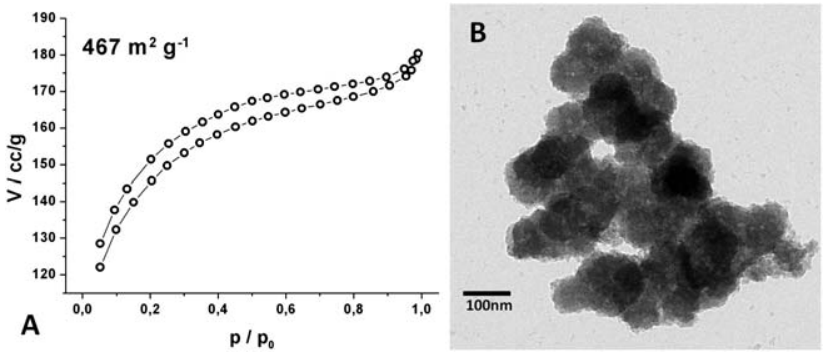

Fig. 11 (A) Nitrogen sorption isotherms of the N-doped carbon after dissolving the silica component. (B) TEM image of the mere carbon (material has been synthesised using a concentration of NHP-dca of $\left.1.6 \mathrm{mmol} \mathrm{L}^{-1}\right)$.

assumed that a mutual stabilisation of the silica and the N-doped carbon components of the material leads to the formation of the observed properties of the composite materials.

\section{Conclusions}

It was shown that the reactivity of nitrogen-doped carbon forming ionic liquids can be easily transferred to other classes of compounds widening the synthetic spectrum in this field of materials chemistry. NHP-dca is a reactive surfactant that can be applied in the sol-gel synthesis of mesoporous silica. Due to its special reactivity a mesoporous composite of silica and nitrogendoped carbon is formed upon annealing under argon. Detailed characterisation of the materials revealed a mesoporous network within submicrometre sized spherical particles, while the porosity can be tuned towards bimodality by varying the concentration of NHP-dca. Further studies on the porosity show that its character seems to be determined by the surfactant only. We were thus able to show an easy one-step approach towards an interesting composite material, showing the potential of our reactive surfactant approach.

\section{Experimental section}

$N$-Hexadecyl-pyridinium-bromide $\quad(\geq 97 \%), \quad$ silver nitrate ( $\geq 99 \%$ ), sodium dicyanamide ( $\geq 96 \%$ ), tetraethylorthosilicate $(\geq 98 \%)$ and methanol $(\geq 98.9 \%)$ were acquired from SigmaAldrich and used without further treatment. Aqueous ammonia solution ( $c=25 \%$, for analysis) was acquired from Carl Roth and used without further treatment.

Annealing of samples was accomplished in a box type furnace (N7/H Nabertherm) equipped with a continuous gas inlet. Thermogravimetric analysis was accomplished using an STA6000 device manufactured by Perkin-Elmer. X-Ray photoelectron spectroscopy (XPS) experiments were performed in a type Theta probe (Thermo Fisher) using monochromatized Al $\mathrm{K}_{\alpha}$ radiation at $h \nu=1486.6 \mathrm{eV}$. Peak positions were internally referenced to the $\mathrm{C} 1 \mathrm{~s}$ peak at $284.6 \mathrm{eV}$. Spectra were deconvoluted using XPSPEAK41 software. Nitrogen sorption isotherms were measured using a Quadrasorb porosimetry device manufactured by Quantachrome, samples were degassed at $150{ }^{\circ} \mathrm{C}$ for $20 \mathrm{~h}$ at reduced pressure before measurement. X-Ray diffraction patterns were measured using $\mathrm{Cu}-\mathrm{K}_{\alpha}$ radiation on a Bruker D8 Advance powder diffractometer. Transmission electron 
micrographs were obtained using a FEI Tecnai $\mathrm{G}^{2} 20$ S-TWIN microscope. Scanning electron micrographs were acquired on a LEO 1550/LEO GmbH Oberkochen provided with an Everhard Thornley secondary electron and In-lens detectors. FT-IR spectra were measured on a Varian 640IR FTIR-spectrometer equipped with a Pike GladiATR device for measurements in attenuated total reflectance mode.

$N$-Hexadecyl-pyridinium-dicyanamide was synthesised by dissolving $15 \mathrm{~g}$ (39 mmol, 1 eq.) of $N$-hexadecyl-pyridiniumbromide in $300 \mathrm{~mL}$ of methanol. Silver dicyanamide was freshly prepared by precipitation mixing equimolar aqueous solutions of sodium dicyanamide and silver nitrate. Silver dicyanamide was filtered, washed with deionised water and directly added in molar excess (1.5 eq.) to the previously prepared solution of $N$-hexadecyl-pyridinium-bromide. The suspension was stirred excluding light for $24 \mathrm{~h}$ before removing the yellow silver bromide/excess silver dicyanamide solid by filtering twice. The product was isolated by removing the solvent at reduced pressure in the rotary evaporator and subsequently dried in high vacuum. $9.86 \mathrm{~g}(27 \mathrm{mmol}, 68 \%)$ of a colourless solid were obtained. Ion exchange could be proven by FT-IR spectroscopy (Fig. S11†).

For the preparation of the mesoporous composite materials of nitrogen-doped carbon and silica, $444 \mathrm{mg}(1.2 \mathrm{mmol})$, $538 \mathrm{mg}(1.4 \mathrm{mmol})$ or $615 \mathrm{mg}(1.6 \mathrm{mmol})$, respectively, of NHP-dca were dissolved in a mixture of $15 \mathrm{~g}$ of deionised water and $1.2 \mathrm{~g}$ of a $25 \%$ aqueous ammonia solution. After the formation of a clear solution $1.25 \mathrm{~g}(6 \mathrm{mmol})$ of tetraethylorthosilicate were added under vigorous stirring. The mixture was stirred for $16 \mathrm{~h}$ before isolation of the colourless silica gel formed upon hydrolysis and condensation of the silica precursor. The gels were annealed in ceramic crucibles under a continuous argon flow of $4 \mathrm{~L} \mathrm{~min}^{-1}$, heating to $1000{ }^{\circ} \mathrm{C}$ with a slope of $\sim 100 \mathrm{~K} \mathrm{~h}^{-1}$ and keeping the samples at $1000{ }^{\circ} \mathrm{C}$ for $1 \mathrm{~h}$. After cooling down to room temperature homogeneous black powders were obtained and pestled for further characterisation. Furthermore calcinations of these composites were carried out by annealing under air at $600{ }^{\circ} \mathrm{C}$ with a heating ramp of $\sim 100 \mathrm{~K} \mathrm{~h}^{-1}$ and subsequently keeping the samples at $600{ }^{\circ} \mathrm{C}$ for $5 \mathrm{~h}$. Additionally silica gels were directly calcined after precipitation at the same heating conditions under air. Both calcination approaches yielded purely white powders that were characterised without further treatment. The silica support of the composite materials was dissolved by stirring in $200 \mathrm{~mL}$ of an aqueous solution of $\mathrm{NH}_{4} \mathrm{HF}_{2}\left(c=4 \mathrm{~mol} \mathrm{~L}^{-1}\right)$ overnight. The black residue was isolated by centrifugation, subsequently washed 5 times with deionised water and dried at $115^{\circ} \mathrm{C}$ in the vacuum drying oven.

\section{Acknowledgements}

Dr Maria-Magdalena Titirici (MPI of Colloids and Interfaces) is kindly thanked for SEM measurements. Sören Selve is gratefully acknowledged for obtaining the TEM micrographs of our materials. Further we would like to thank Dr Silvia Gross (Università di Padova) for her advice regarding XPS data; and Maria Unterweger, Christina Eichenauer and Anne Sobotta for further support of our work. DFG is most gratefully acknowledged for funding (project "Nanocarbon"10033287).

\section{Notes and references}

1 K. P. Gong, F. Du, Z. H. Xia, M. Durstock and L. M. Dai, Science, 2009, 323, 760-764.

2 K. A. Kurak and A. B. Anderson, J. Phys. Chem. C, 2009, 113, 67306734.

3 G. Lalande, R. Cote, D. Guay, J. P. Dodelet, L. T. Weng and P. Bertrand, Electrochim. Acta, 1997, 42, 1379-1388.

4 P. H. Matter and U. S. Ozkan, Catal. Lett., 2006, 109, 115-123.

5 P. H. Matter, L. Zhang and U. S. Ozkan, J. Catal., 2006, 239, 83-96.

6 T. Iijima, K. Suzuki and Y. Matsuda, Synth. Met., 1995, 73, 9-20.

7 Y. P. Wu, S. B. Fang and Y. Y. Jiang, J. Mater. Chem., 1998, 8, $2223-$ 2227.

8 Y. P. Wu, S. B. Fang and Y. Y. Jiang, Solid State Ionics, 1999, 120, $117-123$.

9 Y. P. Wu, C. Y. Jiang, C. R. Wang, S. B. Fang and Y. Y. Jiang, J. Appl. Polym. Sci., 2000, 77, 1735-1741.

10 E. Frackowiak, Phys. Chem. Chem. Phys., 2007, 9, 1774-1785.

11 D. Hulicova, M. Kodama and H. Hatori, Chem. Mater., 2006, 18, 2318-2326.

12 G. Lota, B. Grzyb, H. Machnikowska, J. Machnikowski and E. Frackowiak, Chem. Phys. Lett., 2005, 404, 53-58.

13 G. Lota, K. Lota and E. Frackowiak, Electrochem. Commun., 2007, 9, $1828-1832$.

14 L. Zhao, L. Z. Fan, M. Q. Zhou, H. Guan, S. Y. Qiao, M. Antonietti and M. M. Titirici, Adv. Mater., 2010, 22, 5202-5206.

15 A. Arenillas, T. C. Drage, K. Smith and C. E. Snape, J. Anal. Appl. Pyrolysis, 2005, 74, 298-306.

16 T. C. Drage, A. Arenillas, K. M. Smith, C. Pevida, S. Piippo and C. E. Snape, Fuel, 2007, 86, 22-31.

17 L. Zhao, Z. Bacsik, N. Hedin, W. Wei, Y. H. Sun, M. Antonietti and M. M. Titirici, ChemSusChem, 2010, 3, 840-845.

18 R. Gadiou, A. Didion, R. I. Gearba, D. A. Ivanov, I. Czekaj, R. Kotz and C. Vix-Guterl, J. Phys. Chem. Solids, 2008, 69, 1808-1814.

19 L. Zhao, N. Baccile, S. Gross, Y. J. Zhang, W. Wei, Y. H. Sun, M. Antonietti and M. M. Titirici, Carbon, 2010, 48, 37783787.

20 L. Zhao, R. Crombez, F. P. Caballero, M. Antonietti, J. Texter and M. M. Titirici, Polymer, 2010, 51, 4540-4546.

21 L. J. Zhi, T. Gorelik, R. Friedlein, J. S. Wu, U. Kolb, W. R. Salaneck and K. Mullen, Small, 2005, 1, 798-801.

22 F. Jaouen, M. Lefevre, J. P. Dodelet and M. Cai, J. Phys. Chem. B, 2006, 110, 5553-5558.

23 R. Pietrzak, H. Wachowska and P. Nowicki, Energy Fuels, 2006, 20, $1275-1280$.

24 J. P. Paraknowitsch, A. Thomas and M. Antonietti, J. Mater. Chem., 2010, 20, 6746-6758.

25 J. P. Paraknowitsch, J. Zhang, D. S. Su, A. Thomas and M. Antonietti, Adv. Mater., 2010, 22, 87-92.

26 J. S. Lee, X. Q. Wang, H. M. Luo, G. A. Baker and S. Dai, J. Am. Chem. Soc., 2009, 131, 4596-4597.

27 J. S. Lee, X. Q. Wang, H. M. Luo and S. Dai, Adv. Mater., 2010, 22, 1004-1007.

28 X. Q. Wang and S. Dai, Angew. Chem., Int. Ed., 2010, 49, 6664-6668.

29 J. Y. Yuan, C. Giordano and M. Antonietti, Chem. Mater., 2010, 22, 5003-5012

30 Z. Ma, J. H. Yu and S. Dai, Adv. Mater., 2010, 22, 261-285.

31 D. Carriazo, M. C. Gutierrez, M. L. Ferrer and F. del Monte, Chem. Mater., 2010, 22, 6146-6152.

32 M. C. Gutierrez, F. Rubio and F. del Monte, Chem. Mater., 2010, 22, 2711-2719.

33 Y. Zhou and M. Antonietti, J. Am. Chem. Soc., 2003, 125, 1496014961.

34 T. Nakashima and N. Kimizuka, J. Am. Chem. Soc., 2003, 125, 63866387.

35 Y. Zhou, J. H. Schattka and M. Antonietti, Nano Lett., 2004, 4, 477481.

36 D. Zhang, T. Yan, H. Li and L. Shi, Microporous Mesoporous Mater., 2011, 141, 110-118.

37 M. Antonietti, D. B. Kuang, B. Smarsly and Z. Yong, Angew. Chem., Int. Ed., 2004, 43, 4988-4992.

38 F. Schuth, Angew. Chem., Int. Ed., 2003, 42, 3604-3622.

39 Y. Wan and D. Y. Zhao, Chem. Rev., 2007, 107, 2821-2860.

40 D. Y. Zhao, Q. S. Huo, J. L. Feng, B. F. Chmelka and G. D. Stucky, J. Am. Chem. Soc., 1998, 120, 6024-6036. 
41 A. Fischer, M. Antonietti and A. Thomas, Adv. Mater., 2007, 19, 264 267.

42 A. Fischer, Y. S. Jun, A. Thomas and M. Antonietti, Chem. Mater., 2008, 20, 7383-7389.

43 A. Fischer, J. O. Muller, M. Antonietti and A. Thomas, ACS Nano, 2008, 2, 2489-2496.

44 Y. S. Jun, W. H. Hong, M. Antonietti and A. Thomas, Adv. Mater., 2009, 21, 4270-4274.

45 D. R. MacFarlane, S. A. Forsyth, J. Golding and G. B. Deacon, Green Chem., 2002, 4, 444-448.

46 A. Cros, R. Saoudi, G. Hollinger, C. A. Hewett and S. S. Lau, J. Appl. Phys., 1990, 67, 1826-1830.

47 X-Ray Photoelectron Spectroscopy Database 20, Version 3.0, National Institute of Standards and Technology, Gaithersburg.

48 I. Palchan, M. Crespin, H. Estradeszwarckopf and B. Rousseau, Chem. Phys. Lett., 1989, 157, 321-327.
49 J. A. Taylor, G. M. Lancaster and J. W. Rabalais, Appl. Surf. Sci., 1978, 1, 503-514.

50 S. Delpeux, F. Beguin, R. Benoit, R. Erre, N. Manolova and I. Rashkov, Eur. Polym. J., 1998, 34, 905-915.

51 XPS Database, CNRS, lasurface.com.

52 P. Burg, P. Fydrych, D. Cagniant, G. Nanse, J. Bimer and A. Jankowska, Carbon, 2002, 40, 1521-1531.

53 M. C. Huang and H. S. Teng, Carbon, 2003, 41, 951957.

54 Q. Cai, Z. S. Luo, W. Q. Pang, Y. W. Fan, X. H. Chen and F. Z. Cui, Chem. Mater., 2001, 13, 258-263.

55 M. Grun, I. Lauer and K. K. Unger, Adv. Mater., 1997, 9, 254.

56 H. P. Lin, Y. R. Cheng and C. Y. Mou, Chem. Mater., 1998, 10, 37723776.

57 H. P. Lin and C. Y. Mou, Acc. Chem. Res., 2002, 35, 927-935. 